\title{
Macroscopic chaos in globally coupled maps
}

\author{
M. Cencini ${ }^{(a)}$, M. Falcioni ${ }^{(b)}{ }^{(*)}$, D. Vergni and A. Vulpiani ${ }^{(a)(b)}$ \\ Dipartimento di Fisica, Università "La Sapienza", P.le A. Moro 2, 00185, Roma, Italy \\ (*) Corresponding author (fax:+39-6-4463158; E-mail: M.Falcioni@roma1.infn.it) \\ (a) also INFM, Unità di Roma 1 \\ (b) also INFN, Sezione di Roma 1
}

(June 2, 2018)

\begin{abstract}
We study the coherent dynamics of globally coupled maps showing macroscopic chaos. With this term we indicate the hydrodynamical-like irregular behaviour of some global observables, with typical times much longer than the times related to the evolution of the single (or microscopic) elements of the system. The usual Lyapunov exponent is not able to capture the essential features of this macroscopic phenomenon. Using the recently introduced notion of finite size Lyapunov exponent, we characterize, in a consistent way, these macroscopic behaviours. Basically, at small values of the perturbation we recover the usual (microscopic) Lyapunov exponent, while at larger values a sort of macroscopic Lyapunov exponent emerges, which can be much smaller than the former. A quantitative characterization of the chaotic motion at hydrodynamical level is then possible, even in the absence of the explicit equations for the time evolution of the macroscopic observables.
\end{abstract}

PACS: 05.45.+b

KEYWORDS: Macroscopically coherent dynamics, Finite size Lyapunov exponent, Globally coupled maps 


\section{INTRODUCTION}

In the last years the emergence of non trivial collective behaviour in high-dimensional dynamical systems has gathered much attention [1 \&]. An interesting limit case of macroscopic coherence is the global synchronization of all the parts of the system: this behaviour has been found in many different situations in physics, chemistry and biology. Relevant examples of synchronization are the flickering of swarms of fireflies, fish locomotion, Josephson arrays and certain multi-mode laser systems [9].

Beyond synchronization there exist much more interesting and intriguing phenomena involving non trivial coherence. Let us mention the relevant works of Kaneko [2] showing the emergence of subtle coherence among different elements of globally coupled maps (GCM), in the case of a logistic local map.

A GCM system (of the type considered in this paper) is a collection of elements $x_{n}$ (defined on the site $n$ ) evolving according to the equation:

$$
x_{n}(t+1)=(1-\epsilon) f_{a}\left(x_{n}(t)\right)+\frac{\epsilon}{N} \sum_{i=1}^{N} f_{a}\left(x_{i}(t)\right),
$$

where $N$ is the total number of elements, $t$ is the discrete time and $f_{a}(x)$ is a nonlinear function depending on a parameter $a$.

When the mapping function is $f_{a}(x)=a x(1-x)$ (i.e. a logistic map), for certain values of $\epsilon$ and $a$ one can observe rather remarkable behaviours, as anomalous fluctuations, glassy behaviour and broken ergodicity [3]. For instance, Kaneko [2] studied a parameters region where the number of positive Lyapunov exponents is proportional to $N$; in this region the local elements $x_{n}$ take different values, almost randomly, so one might expect that the temporal fluctuations of the mean-field

$$
h(t)=\frac{1}{N} \sum_{i=1}^{N} f_{a}\left(x_{i}(t)\right)
$$

obey a Gaussian statistics, with a mean square deviation $\sigma_{N}^{2}=\left\langle h^{2}\right\rangle-\langle h\rangle^{2}$ proportional to $N^{-1}$. On the contrary one observes $\sigma_{N}^{2} \rightarrow$ const, as $N \rightarrow \infty$. Only with the inclusion of noise one obtains $\sigma_{N}^{2} \sim N^{-\beta}$, with $\beta<1$, if the noise is not too strong. This is due to a coherence (collective behaviour) among the elements $x_{n}$, that can be associated [2] with the existence of the periodic windows in the chaotic region of the logistic map.

In some regions of the parameters space one observes clustering [4], i.e., the elements $x_{n}$ split into clusters, such that the elements belonging to the same cluster $k$ take an identical value $\tilde{x}_{k}(t)$. The number $\mathcal{C}$ of the possible clusters can be very large with $N(\ln \mathcal{C} \sim \sqrt{N})$ and the dynamics can show glass-like behaviour and failure of ergodicity [3].

Symplectic GCM, where the coupling is not of the mean-field type of eq. (1), can show a more intriguing behaviour for the clustered motion, since, in this case, the synchronization between the different parts is not perfect and the clustering has a finite lifetime [5].

Even coupled map lattices (CML) with local coupling, on $d$-dimensional lattices, can show a macroscopic behaviour that is very different from the microscopic one. For example, Chaté and Manneville [1] showed that in CML on hyper-cubic $d$-dimensional lattices some macroscopic variables, as the center of mass: 


$$
m(t)=\frac{1}{N} \sum_{i=1}^{N} x_{i}(t),
$$

can have periodic behaviour, or quasi-periodic for $d \geq 5$, even if the system is chaotic, i.e. the first Lyapunov exponent is positive.

In our opinion, one of the more interesting facts among the many different aspects of the high-dimensional dynamical systems is the macroscopic chaos. With this locution we indicate the irregular behaviour of some global variables (e.g. the center of mass) that evolve with typical times much longer than the characteristic time of the full dynamics, that we call the microscopic dynamics; the order of magnitude of the latter time may be estimated by the inverse of the first Lyapunov exponent $\lambda_{1}$ of the microscopic dynamics

The macroscopic chaos for the high-dimensional maps, in some sense, is the analogous of the hydrodynamical chaos for the molecular motion. Let us briefly clarify this point and stress the open problems. For the Hamiltonian dynamical system describing the evolution of the molecules of a fluid the order of magnitude of the microscopic characteristic time is the mean collision time. On the other hand, if one studies the hydrodynamic equations of the considered fluid - those describing the motion on space and time scales that are very large with respect to the molecular scales - the involved characteristic times are much larger. So one expects that for the full Hamiltonian dynamics $\lambda_{1} \sim 10^{11} \mathrm{~s}^{-1}$, while the first Lyapunov exponent for the hydrodynamic equations, in a chaotic regime, is of order $\left(10^{-1}-1\right) \mathrm{s}^{-1}$, the exact value depending on the geometry of the system, the external forcing, the Reynolds number and so on.

This big difference comes from the coarse graining process in the building of the hydrodynamic equations, that is from the very different scales one is observing in the two cases.

If one is able to write down the equations at the hydrodynamical level (i.e. for the slow variables) then it is not difficult to characterize the macroscopic behaviour, by means of the standard techniques of dynamical system theory. However in this case, one subtle point, on the mathematical side, is the problem of the norm to be used in the definition of the Lyapunov exponents. For finite dimensional systems all the norms are equivalent [10] and there is no ambiguity. This is not true for infinite dimensional systems (as the hydrodynamical partial differential equations are), so that the Lyapunov exponents can depend on the used norm.

In generic CML, as far as we know, there are no general systematic methods to build up the macroscopic equations; therefore it is necessary to study the macroscopic behaviour of the system in terms of the $x_{n}$ variables, i.e. it is necessary to remain at the microscopic level of description.

The present paper is organized as follows. In the next section we report some phenomenology of macroscopic chaos in GCM. Section III is devoted to a discussion on the recently proposed self-consistent Perron-Frobenius method for the one-body description of these systems at a macroscopic level. In section IV, that is the nucleus of the paper, we show that the recently introduced finite size Lyapunov exponent gives a suitable quantitative characterization of the macroscopic chaos; that is to say: we are able to identify a macroscopic characteristic time by means of a Lyapunov exponent that is effective on the macroscopic scales and which, in general, is much smaller than the usually defined one. The last section is devoted to discussion and conclusions. In the Appendix we discuss some subtle points 
related to the problem of the choice of the norm in the definition of the Lyapunov exponent for the one-body description.

\section{SOME PHENOMENOLOGICAL RESULTS AND FIRST NAIVE APPROACHES}

For sake of self-consistency, we report some numerical evidence (partly already known from previous works [2, 6, (7]) of the existence of macroscopic chaos in GCM, eq. (11). For a detailed description of the many different macroscopic behaviours see the works of Kaneko [2,77] and also Pikovsky and Kurths [6].

We studied mainly systems (11) of globally coupled tent maps, i.e.: $f_{a}(x)=a(1 / 2-$ $|x-1 / 2|)$, and all the figures of this paper refer to these systems. In addition, to test the validity of our method, we also considered a slightly different version of eq. (11), the so-called heterogeneous GCM [7], in which the interacting elements are not all identical:

$$
x_{n}(t+1)=(1-\epsilon) f_{a_{n}}\left(x_{n}(t)\right)+\frac{\epsilon}{N} \sum_{i=1}^{N} f_{a_{i}}\left(x_{i}(t)\right),
$$

the control parameters $a_{i}$ are quenched variables that take values according to some rule to be specified. Here we chose $f_{a_{i}}(x)=1-a_{i} x^{2}$ (the logistic map), with $a_{i}$ assuming equally spaced values in an interval between $a_{\min }$ and $a_{\max }$.

At varying $\epsilon$ and $a$ in eq. (四) or $a_{\min }$ and $a_{\max }$, in eq. (雨), and by looking at the evolution of a macroscopic variable as, for instance, $m(t)$, we observed different macroscopic behaviours, which can be grouped in the three following classes.

i) STANDARD CHAOS: the microscopic Lyapunov exponent is positive and the law of large numbers holds. In this case, if one plots $m(t)$ versus $m(t-1)$, one observes a spot with size of order $1 / \sqrt{N}$.

ii) MACROSCOPIC PERIODICITY: the microscopic Lyapunov exponent is positive and the plot of $m(t)$ versus $m(t-1)$ gives rise to $k$ spots with size of order $1 / \sqrt{N}$, for a macroscopic motion of period $k$. There are also less trivial periodic or quasi-periodic behaviours (e.g. in [7] a case is shown in which the mean-field dynamics evolves on a 2-dimensional torus).

iii) MACROSCOPIC CHAOS: the microscopic Lyapunov exponent is positive and the plot of $m(t)$ versus $m(t-1)$ shows an apparently well structurated function, see fig. 1, that suggests chaotic motion for $m(t)$.

The first hope is that, at a macroscopic level, the system can be described by few global variables, that are able to catch the large scale properties, i.e. one hopes that the macroscopic motion is driven by a low dimensional dynamics. Indeed, fig. 1a (that is obtained for particular parameters values of the tent GCM), seems to suggest such a possibility: the first-return plot for the center of mass evolution appears to generate a one dimensional map. In such a case one can conjecture that

$$
m(t+1)=F(m(t))+\eta_{N}(t)
$$


where $\eta_{N}$ is a small $(\sim 1 / \sqrt{N})$ incoherent fluctuation, vanishing in the limit $N \rightarrow \infty$. If the above equation holds - with the evolution function $F(m)$ fitted from the data - one can define the Lyapunov exponent for dynamical system (5). This latter quantity gives information on the sensibility of the evolution of the macroscopic variable $m$ on the initial conditions, so that one may call it macroscopic Lyapunov exponent $\lambda_{\text {macro }}$; according to the standard definition, one has:

$$
\lambda_{\text {macro }}=\int \ln \left|F^{\prime}(m)\right| P(m) d m,
$$

where $P(m)$ is the probability distribution of $m$, as obtained from numerical simulations of eq. (1). In the case of macroscopic chaos one expects that $\lambda_{\text {macro }}>0$. On the contrary, using the recipe of eq. (6) one obtains that $\lambda_{\text {macro }}$ is negative. This result is not a paradox: simply eq. (5) does not hold. If one looks carefully at the plot of $m(t)$ versus $m(t-1)$, for large $N$, one realizes that very fine grained structures exist, see fig. $1 \mathrm{~b}$. This suggests that the variable $m(t)$ alone is not sufficient for a satisfying description of the macroscopic properties of the system. Therefore we need to introduce some other variables. It seems likely that the system evolution at a macroscopic scale is driven by a full set of equations of phenomenological type, such as, e.g.:

$$
\begin{cases}m(t+1)= & F_{1}(m(t), \sigma(t), \ldots)+\eta_{N}^{(1)} \\ \sigma(t+1)= & F_{2}(m(t), \sigma(t), \ldots)+\eta_{N}^{(2)} \\ \vdots & \vdots\end{cases}
$$

where $\sigma^{2}=\left(\sum x_{i}^{2}\right) / N-\left(\sum x_{i} / N\right)^{2}$, and $\eta_{N}^{(i)}$ are small fluctuations vanishing as $N \rightarrow \infty$. Unfortunately, as far as we know, there is not a systematic approach (even of numerical type) for the building of eq.s (耳), i.e. for the determination of the functions $F_{1}, F_{2}, \ldots$.

Another naive approach, based on the possible time scales separation between the microscopic and the macroscopic dynamics, is the analysis of the microscopic and macroscopic time correlation functions, defined as follows:

$$
\begin{aligned}
C_{\text {micro }}^{(i)}(\tau) & =\frac{\left\langle x_{i}(t+\tau) x_{i}(t)\right\rangle-\left\langle x_{i}\right\rangle^{2}}{\left\langle x_{i}^{2}\right\rangle-\left\langle x_{i}\right\rangle^{2}} \\
C_{\text {macro }}(\tau) & =\frac{\langle m(t+\tau) m(t)\rangle-\langle m\rangle^{2}}{\left\langle m^{2}\right\rangle-\langle m\rangle^{2}},
\end{aligned}
$$

where $\langle(\ldots)\rangle$ means time average along the trajectory.

It is to be noted, at this point, that the system we are studying shows a non trivial macroscopic behaviour in a parameter region where it is also in a broken ergodicity phase. In fig. 2 we show the probability distributions, $P_{n}(x)$, of two different $x_{n}$ for the system (11) in a situation of macroscopic chaos. It is well evident that the knowledge of the probability distribution of a generic $x_{n}$ does not permit a good description of the system (this is what we mean, in such a setting, by saying that the system is not ergodic). It is important to note that the two elements have been chosen in the two different bands in which the $\left\{x_{n}\right\}$ are grouped, see [2]. Moreover elements belonging to the same band seems to have the 
same distributions. Let us stress that this behaviour is not related to the particular band structure of the tent GCM: we found non trivial macroscopic motion in the presence of broken ergodicity also in heterogeneous GCM (田), where the band structure is absent, but the microscopic building elements are not identical.

Fig. 3 shows the above defined correlation functions in the regime of macroscopic chaos. Since one has a failure of the ergodicity, different sites have different autocorrelations, that is $C_{\text {micro }}^{(i)}(\tau)$ depends on the band to which the element $x_{i}$ belongs. Rather unexpected is the fact that the characteristic decay times of $C_{m i c r o}^{(i)}(\tau), \tau_{m}^{i}$, and of $C_{\text {macro }}(\tau), \tau_{M}$, defined by the equations

$$
C_{\text {micro }}^{i}(\tau) \sim e^{-\tau / \tau_{m}^{i}}, \quad C_{\text {macro }}(\tau) \sim e^{-\tau / \tau_{M}},
$$

are not very different; for instance, in the case of fig. 3 one has: $\tau_{m}^{i} \approx \tau_{M} \approx 115$. A non trivial point is that these typical decay times are much larger than the characteristic time given by the Lyapunov exponent, i.e. $1 / \lambda_{1}$ (for the system of fig. 3 one has $1 / \lambda_{1} \approx 4$.). This fact can be related to the strong intermittency of the system. We have to stress that this behaviour of the correlation times has been observed also in the heterogeneous logistic GCM (4).

In the next sections we discuss some less naive approaches to the characterization of the macroscopic chaos.

\section{ONE-BODY DESCRIPTION IN TERMS OF A SUITABLE PERRON-FROBENIUS OPERATOR}

Let us now discuss how one can try to characterize the coherent dynamics of GCM in terms of a suitable Perron-Frobenius (PF) operator [2,6,8]. At any time $t$ one can wonder about the snapshot density $\rho_{t}(y)$, defined by the probability density that $x_{n}(t)$ assumes the value $y$ :

$$
\rho_{t}(y)=\frac{1}{N} \sum_{i=1}^{N} \delta\left(y-x_{i}(t)\right)
$$

Using the fact that, for a mean field coupling as in eq. (1), each $x_{n}$ interacts with all the other $\left\{x_{i}\right\}$ in the same way, the time evolution of $\rho_{t}(y)$ can be written in terms of $\rho_{t-1}(y)$, by means of a self-consistent approach:

$$
\rho_{t}(y)=\mathcal{L}^{(1)} \rho_{t-1}(y)=\frac{1}{1-\epsilon} \sum_{i} \frac{\rho_{t-1}\left(y_{i}\right)}{\left|f_{a}^{\prime}\left(y_{i}\right)\right|},
$$

where the sum is on the preimages given by

$$
y_{i}=f_{a}^{-1}\left(\frac{y-\epsilon \int f_{a}(z) \rho_{t-1}(z) d z}{1-\epsilon}\right),
$$

$f_{a}^{-1}$ being the inverse function of $f_{a}$, for instance, if $f_{a}(y)=1-a y^{2}$ then $f_{a}^{-1}(z)=$ $\pm \sqrt{(1-z) / a}$. 
In the parameter region of the standard chaos (see sect. 【I) where $m(t)=$ const. + $O(1 / \sqrt{N})$, one has that, after long times, $\rho_{t}(y)$ converges toward a stationary distribution $\rho_{\text {eq }}(y)$, i.e.:

$$
\rho_{t}(y) \rightarrow \rho_{e q}(y) \quad \text { for } \quad t \rightarrow \infty \text {. }
$$

On the other hand, for a macroscopic periodic behaviour, the long time properties of the density $\rho_{t}(y)$ are described by means of a finite collection of functions of $y$; e.g. when $m(t)$ asymptotically oscillates among two different values:

$$
m(2 t)=m_{e} \quad m(2 t+1)=m_{o}
$$

after a transient time one observes

$$
\rho_{2 t}(y)=\rho_{e}(y) \quad \rho_{2 t+1}(y)=\rho_{o}(y)
$$

Finally, in the case of macroscopic chaos $\rho_{t}(y)$ does not converge toward any finite set of function of $y$, but evolves following a nontrivial time behaviour.

The above approach for $\rho_{t}(y)$ has been introduced by Perez and Cerdeira [ 8 ] and K. Kaneko [2]. From a conceptual point of view eq.s (12 - 13) are close to the Boltzmann equation for the gases [11]. Indeed, as one can check by a direct inspection, the PF operator $\mathcal{L}^{(N)}$ that rules the evolution of the probability density $\rho_{t}^{(N)}\left(y_{1}, y_{2}, \ldots, y_{N}\right)$ in the whole phase space:

$$
\rho_{t}^{(N)}\left(x_{1}, x_{2}, \ldots, x_{N}\right)=\mathcal{L}^{(N)} \rho_{t-1}^{(N)}\left(x_{1}, x_{2}, \ldots, x_{N}\right),
$$

is a linear operator. On the other hand the PF operator $\mathcal{L}^{(1)}$ for the evolution of $\rho_{t}(y)$, given by eq.s (12 - 13), is a nonlinear one [6], since it depends explicitly on $\rho_{t-1}(y)$. In the kinetic theory of gases one has a similar situation: the Liouville equation, for the evolution of the probability density in the whole phase space, is linear, while the Boltzmann equation for the one-body statistics is nonlinear.

Since the evolution equations (12 - 13) for $\rho_{t}(y)$ are a dynamical system (though of infinite dimension), one can formally introduce the notion of first Lyapunov exponent [2]. By so doing, as noted in the introduction, one is faced with the mathematical problem of the choice of the norm: for sake of simplicity we adopt the usual norm in the $L_{2}$ space. One could think that the problem of the norm is relevant just for strictly mathematical reasons, while from a physical point of view this problem practically does not exist. In the Appendix we discuss how even for very simple shapes of $\rho(x)$ one obtains different Lyapunov exponents using different norms.

However to practically obtain the first Lyapunov exponent, firstly one applies an infinitesimal perturbation $\delta \rho_{0}(y)$ on $\rho_{t}(y)$ (in order to satisfy the normalization condition one has to impose the constraint $\left.\int \delta \rho(y) d y=0\right)$ then one observes the evolution $\delta \rho_{n}(y)$ of the perturbation. In this way, following the standard methods of Benettin et al. [12], it is possible to estimate the whole set of the Lyapunov exponents $\left\{\tilde{\lambda}_{i}\right\}$; in particular one has

$$
\tilde{\lambda}_{1}=\frac{1}{2} \lim _{T \rightarrow \infty} \lim _{\delta \rho_{0} \rightarrow 0} \frac{1}{T} \sum_{n=0}^{T-1} \ln \frac{\int\left|\delta \rho_{n+1}(y)\right|^{2} d y}{\int\left|\delta \rho_{n}(y)\right|^{2} d y} .
$$


It is easy to understand that $\tilde{\lambda}_{1}$ can give interesting "global information" which is completely different from the one given by the microscopic Lyapunov exponent. In the case of standard chaos, or macroscopic periodic behaviour, $\tilde{\lambda}_{1}$ is negative, or zero, while for macroscopic chaos $\tilde{\lambda}_{1}>0$. Let us stress that $\tilde{\lambda}_{1}$ can be negative even if the first Lyapunov exponent for the dynamical equations (1) is positive. Some detailed numerical investigation of Kaneko on GCM show that $\tilde{\lambda}_{1}$ is positive in the cases of macroscopic chaos [2].

Equations (12 - 13) hold only in the case of a mean field interaction for homogeneous GCM (eq. 1) and other particular systems [6], but they are not valid even for the heterogeneous GCM (eq. \#). Nevertheless, the computation of $\tilde{\lambda}_{1}$ according to the definition (18), remains possible using a numerical approach - at least in principle, even if it may result in a very huge and heavy task - also for generic CML (i.e., in the case of maps with local coupling). This is accomplished by following the evolution of $\rho_{t}(y)$, as given by the time development of the histogram of the instantaneous microscopic configuration $\left(x_{1}, x_{2}, \ldots, x_{N}\right)$.

Besides the previous relevant properties of $\tilde{\lambda}_{1}$, we have to underline a delicate point that rises for its numerical computation, when it is not possible to write down the appropriate eq. (12). In this case, as we already pointed, one is forced to study the evolution of $\left\|\delta \rho_{t}(y)\right\|$ making histograms from two instantaneous configurations $\left\{x_{i}\right\}$ and $\left\{x_{i}^{\prime}\right\}$, obtained by the introduction of a small perturbation on $\left\{x_{i}\right\}$ at $t=0$. To show where is the problem, let us consider the time evolution of $\delta m(t)$ in the case of standard chaos. One can write $\delta m$ in two different ways:

$$
\begin{aligned}
& \delta m(t)=\frac{1}{N} \sum_{n=1}^{N} \delta x_{n}(t) \\
& \delta m(t)=\int y \delta \rho_{t}(y) d y .
\end{aligned}
$$

If we are in the parameters region where the Lyapunov exponent $\lambda_{1}$ is positive, but $\tilde{\lambda}_{1}$ is negative, we have both $\left|\delta x_{n}(t)\right| \sim\left|\delta x_{n}(0)\right| \exp \left(\lambda_{1} t\right)$ and $\left\|\delta \rho_{t}|| \sim\right\| \delta \rho_{0}|| \exp \left(-\left|\tilde{\lambda}_{1}\right| t\right)$. So that apparently we fall into a contradiction, since from (19) we obtain

$$
|\delta m(t)| \sim e^{\lambda_{1} t}
$$

while from (20) we have

$$
|\delta m(t)| \sim e^{-\left|\tilde{\lambda}_{1}\right| t}
$$

To eliminate the contradiction one needs to take into proper account the scales of the perturbations on which these two equations hold true; eq. (21) holds at small scales: $|\delta m| \ll$ $O(1 / \sqrt{N})$, while eq. (22) holds for $|\delta m| \gg O(1 / \sqrt{N})$. Basically if one starts with a perturbation $\delta m(0)$ much smaller than $O(1 / \sqrt{N})$ one has an exponential growth, but only up to a magnitude $O(1 / \sqrt{N})$, the maximum allowed by the laws of large numbers. In an analogous manner starting from a perturbation $\delta m(0)$ much larger than $O(1 / \sqrt{N})$ one has an exponential decrease up to $O(1 / \sqrt{N})$, see fig. 4 .

From the previous example it is clear that if one studies numerically the time evolution of $\left\|\delta \rho_{t}\right\|$ for large but finite $N$, at small times (or, more precisely, small $\|\delta \rho\|$ ) one can observe 
just the "microscopic" Lyapunov exponent. So one has to introduce some coarse-graining i.e. to consider non infinitesimal $\delta \rho_{0}$ - to obtain a macroscopic level description. However, it must be noted that there is a large arbitrariness in choosing a non infinitesimal $\delta \rho_{0}$.

In this section we have discussed the computation of the macroscopic Lyapunov exponent by means of the Perron-Frobenius method, both in its original formulation eqs. (12- 13) and in its numerical version, to be applied when the P-F equation cannot be explicitly written. Neither approach is easy to handle because of the arbitrariness in the choice of the norm and of the non infinitesimal $\delta \rho_{0}$.

In order to avoid these difficulties (numerical and conceptual) concerning the one-body description, in the next section we introduce a different approach.

\section{CHARACTERIZATION OF THE MACROSCOPIC CHAOS VIA THE PREDICTABILITY IN THE LARGE}

The introduction of efficient methods (Benettin et al. [12]) for the computation of the Lyapunov exponents has been an important milestone in the understanding of chaotic systems. However, in spite of their deep conceptual relevance, the Lyapunov exponents are not able to give a complete characterization of realistic dynamical systems, such as systems with intermittent behaviour or with many different characteristic times. For example, in order to describe intermittency effects one needs to introduce generalized Lyapunov exponents and the statistics of the fluctuations of the effective Lyapunov exponent 13 15]. On the other hand in systems with many different characteristic times (e.g. the fully developed turbulence) the Lyapunov exponent cannot be used to study, even at very rough level, the predictability problem for small (but not infinitesimal) perturbations, that cannot be described in terms of the tangent vector. In order to overcome these difficulties recently a new indicator has been introduced: the finite size Lyapunov exponent (FSLE), see Ref. [16] for details.

The definition of FSLE may be given in terms of the predictability time $T_{r}(\delta)$, that is the time a perturbation of initial size $\delta$ takes to grow by a factor $r(>1)$ during the system evolution. The perturbation of size $\delta$ is supposed to be already aligned with the most unstable direction. The factor $r$ should be taken not too large, in order to avoid the growth trough different scales. In many applications, $r=2$, so that sometimes the $T_{r}(\delta)$ is also called the error doubling time. The Finite Size Lyapunov Exponent is defined from a suitable average of the predictability time according to

$$
\lambda(\delta)=\left\langle\frac{1}{T_{r}(\delta)}\right\rangle_{\mathcal{N}} \ln r=\frac{1}{\left\langle T_{r}(\delta)\right\rangle} \ln r
$$

where $\langle\cdots\rangle_{\mathcal{N}}$ denotes average with the natural measure along the trajectory and $\langle\cdots\rangle$ is the average over many realizations, for more details see ref. [17]. The above definition holds for continuous time system. In the case of discrete time systems (i.e. maps) $T_{r}(\delta)$ is the minimum time such that the distance $\Delta_{r}$ between the two realizations is greater or equal to $r \delta$. Therefore, instead of eq. (23) we have [17]:

$$
\lambda(\delta)=\frac{1}{\left\langle T_{r}(\delta)\right\rangle}\left\langle\ln \left(\frac{\Delta_{r}}{\delta}\right)\right\rangle .
$$


Let us note that by the definition (23) $\lambda(\delta)$ is not appropriate in discerning cases with $\lambda=0$ and $\lambda<0$, due to the positiveness of the predictability time.

For chaotic systems the limit of infinitesimal perturbations $\delta \rightarrow 0$ in (23) gives the maximum Lyapunov exponent $\lambda_{1}$, i.e. $\lambda(\delta)$ displays a plateau at the value $\lambda_{1}$ for sufficiently small $\delta$.

In many realistic situations, the error growth for infinitesimal perturbations is dominated by the faster scales, that are typically the smaller ones (as in the classic example of three dimensional turbulent flows). When the size $\delta$ of the perturbation cannot be considered any longer infinitesimal, the behaviour of $\lambda(\delta)$ is governed by the nonlinear evolution of the perturbation, and, in general, $\lambda(\delta)<\lambda_{1}$. The decrease of $\lambda(\delta)$ does follow a systemdependent law. In some cases, $\lambda(\delta)$ can be predicted by dimensional arguments, e.g. in the fully developed turbulence one has the universal law $\lambda(\delta) \sim \delta^{-2}$, in the inertial range [16, 17].

Therefore, the behaviour of $\lambda$ as a function of $\delta$ gives important information on the characteristic times (and scales [18]) governing the system and is a powerful tool in studying dynamical systems which involve many characteristic scales in space and time.

The FSLE has been successfully used even in time series analysis [19]. A relevant practical fact is that, at variance with the usual Lyapunov exponent which needs high embedding dimensions and extremely huge statistic, the FSLE at relatively large $\delta$ can be extracted even using moderately small embedding dimension. For example, in systems in which some slow variables can be separated from the fast variables, the FSLE related to the slow dynamics can be obtained even with moderate statistics and unresolved small scales (i.e. the fast ones).

To practically compute the FSLE, firstly one has to define a series of thresholds $\delta_{n}=r^{n} \delta_{0}$ $(n=1, \ldots, M)$, and to measure the times $T_{r}\left(\delta_{n}\right)$ that a perturbation of size $\delta_{n}$ takes to grow up to $\delta_{n+1}$. The times $T_{r}\left(\delta_{n}\right)$ are obtained by following the evolution of a perturbation from its initial size $\delta_{\min }<\delta_{0}$ up to the largest threshold $\delta_{M}$. This can be done by integrating two trajectories of the system starting at an initial distance $\delta_{\min }$. The FSLE, $\lambda\left(\delta_{n}\right)$, is then computed by averaging the predictability times over several realizations, see equation (24). In general, one must choose $\delta_{\min } \ll \delta_{0}$, in order to allow the direction of the initial perturbation to align with the most unstable direction in the phase-space. Moreover, one must pay attention to keep $\delta_{M}<\delta_{\text {saturation, }}$, so that all the thresholds can be attained.

Our hope is that, as in the usual hydrodynamical description of thermodynamic systems, the variables of systems with macroscopic chaos can be separated in slow variables (given by $m(t), \sigma(t)$ and so on) and fast variables.

We have computed the FSLE (23) looking only at $m(t)$ both for the homogeneous GCM (回), using as local map the tent map, and for the heterogeneous GCM (田), for different values of the parameters. Practically the numerical experiments has been performed as follows: we have averaged, over many realizations, the predictability times $T_{r}\left(\delta_{n}\right)$ (defined above) related to $|\delta m(t)|$ which is initialized at the value $\delta m(t)=\delta_{m i n}$. This has been done considering, for each realization, the perturbed system that is obtained by shifting all the elements of the unperturbed one by the quantity $\delta_{\min }$ (i.e. $\left.x_{i}^{\prime}(0)=x_{i}(0)+\delta_{\min }\right)$.

As can be seen from figure $5 \mathrm{a}$, which shows $\lambda(\delta)$ versus $\delta$ in the case with macroscopic chaos, two plateaux exist: at small values of $\delta\left(\delta \leq \delta_{1}\right)$ one has, as expected from general considerations, $\lambda(\delta)=\lambda_{1}$; for $\delta \geq \delta_{2}$ one has another plateau which can fairly be called "macroscopic" Lyapunov exponent, $\lambda(\delta)=\lambda_{\text {macro }}$. Moreover, $\delta_{1}$ and $\delta_{2}$ decrease at increas- 
ing $N$. In fact, from figure $5 \mathrm{~b}$, where we report $\lambda(\delta)$ versus $\delta \sqrt{N}$ for different values of $N$, one can see that both $\delta_{1}$ and $\delta_{2}$ scale as $\sim 1 / \sqrt{N}$ : by rescaling $\delta$ with $\sqrt{N}$, one observes that $\delta_{1} \sqrt{N}$ (which indicates the end of the microscopic plateau) and $\delta_{2} \sqrt{N}$ (which marks the end of the saturation due to the microscopic dynamics and the beginning of the macroscopic plateau) coincide for all values of $N$. Incidentally, one can also observe that the macroscopic plateau, being almost non-existent for $N=10^{4}$, becomes more and more resolved and extended on large values of $\delta \sqrt{N}$ at increasing $N$ up to $N=10^{7}$. From these features we can argue that the macroscopic motion is well defined in the limit $N \rightarrow \infty$ and therefore we can conjecture that in this limit the microscopic signature in the evolution of $\delta m(t)$ completely disappears in favor of the macroscopic behaviour.

It is important to note that in order to obtain the results shown in fig. 5 and fig. 7 some precautions must be taken in initializing the system. In fact, because of the complex structure of the phase space of this system, one has that different initial conditions can led to different attractors [2]. Therefore, choosing random initial conditions while increasing $N$, one likely obtains different values for the macroscopic and/or microscopic Lyapunov exponents. In order to maintain the system on the same attractor, we kept fixed the percentage of elements per band (see Kaneko [2]), at varying the number of elements $N$.

In the case of standard chaos $\left(\lambda_{\text {macro }}<0\right)$ one has only the microscopic plateau and then a fast decreasing of $\lambda(\delta)$, as can be seen in fig. 6. Also in the cases of non trivial macroscopic behaviour, either periodic or quasi-periodic (i.e. $\lambda_{\text {macro }}=0$ ), we see only the microscopic plateau followed by a decreasing up to the saturation. This is a consequence of the fact that $\lambda(\delta)$ is not able to distinguish between $\lambda<0$ and $\lambda=0$, by definition, see eq. (23).

One can wonder if it is possible to compute the macroscopic Lyapunov exponent directly looking at $\langle\ln |\delta m|\rangle$ versus $t$. The macroscopic regime is expected to appear as a change of slope when the size of the perturbation is $O(1 / \sqrt{N}$ ) (as discussed in sect. III in the case of standard chaos). In fig. $7\langle\ln |\delta m|\rangle$ is shown as a function of $t$ for the same systems of fig. 5 (with identical initial conditions). One can see that for small values of $\delta m(t)$ one has a well defined exponential growth according to the microscopic Lyapunov exponent. This regime stops at $\delta m(t) \sim O(1 / \sqrt{N})$ i.e. when the microscopic error growth saturates. At larger values of $\delta m(t)$ one observes a second (macroscopic) slope. One has a clear evidence also from fig. 7 that the macroscopic motion sets in at a scale that is smaller when $N$ is larger, as already established by the analysis of fig. 5. In the case of fig. 7 one has also a fair quantitative agreement with the results obtained with the FSLE method (fig. 5). However in general, because of the intermittency, the macroscopic regime can start at different times and with different rates, therefore the naive method in which the averages are performed at fixed delay times can give spurious laws; see ref [16,18] for a discussion of these effects.

Of course a priori there is no deep reason to believe that the above analysis performed only on one variable, $m(t)$, is enough accurate, therefore we performed a similar study looking at two macroscopic variables, $m(t)$ and $\sigma(t)$. Since there is not a natural way to introduce a perturbation on $m(t)$ and $\sigma(t)$, we are forced to follow the method of Wolf et al. [20] for the computation of the Lyapunov exponent from data, but with some modifications. We considered the phase space generated by the variables $m(t), \sigma(t)$ and we looked at the time separation between analogues whose initial distance $\delta_{0}$ is not very small (i.e. in the region of the "macroscopic" plateau in fig. 5). Following the evolution of the full system we compute the quantity: 


$$
\Gamma(\tau)=\frac{1}{\mathcal{A}} \sum_{j=1}^{\mathcal{A}} \ln \frac{\Delta\left(t_{j}+\tau\right)}{\Delta\left(t_{j}\right)},
$$

where $\Delta(t)=\sqrt{\delta m^{2}(t)+\delta \sigma^{2}(t)}, t_{j}$ are the "return" times such that $\Delta\left(t_{j}\right) \in\left[\delta_{0}, 2 \delta_{0}\right]$ and $\mathcal{A}$ is the number of analogues. Fig. 8 shows $\Gamma(\tau)$ versus $\tau$.

It is important to stress the relevance of the value of $\delta_{0}$, which must be not too small. Using a $\delta_{0}$ out of the "macroscopic" (i.e. $\delta_{0}<\delta_{2}$ ) plateau one has spurious behaviour due to intermittency (see ref. [18]).

It is remarkable the agreement between the values for the macroscopic Lyapunov exponent obtained with the two methods.

\section{CONCLUSION AND DISCUSSION}

In this paper we proposed a quantitative characterization of the coherent evolution in globally coupled maps that show macroscopic chaos. From a conceptual point of view, the irregular behaviours of global observables - which typically evolve on time scales much longer than those related to the evolution of a single element $x_{n}(t)$ - are for the GCM the analogous of the chaos for hydrodynamical equations in the description of the molecular motion.

In generic systems there are not a systematic method to obtain the equations that describe the evolution of the degrees of freedom accounting for the large scale behaviour. Therefore a straightforward application of the usual dynamical systems methods is not able to give suitable characterizations. In particular, the usual Lyapunov exponent, which gives a microscopic description, is not able to capture the essential features of this macroscopic phenomenon.

It is worth to note that in the cases where it is possible to derive hydrodynamical-like equations, such as the Perron-Frobenius equation, one obtains a description of the system at a macroscopic level, see for a detailed discussion [2,6]. We have shown that, in the cases in which this approach is missing, the recently introduced Finite Size Lyapunov Exponent |16] is able to characterize, in a consistent way, the macroscopic behaviour of the system and gives a measure of the chaotic motion at a coarse-grained level. We have the following scale-dependent scenario for the mechanism acting on the evolution of the perturbation of global observables (i.e. the mean field):

- at small values of the perturbation $(\ll 1 / \sqrt{N})$ we recover the microscopic Lyapunov exponent, simply because at these scales the evolution of the perturbation is driven by the microscopic dynamics;

- at larger values $(\gg 1 / \sqrt{N})$ we obtain a macroscopic Lyapunov exponent, which can be much smaller than the first Lyapunov exponent of the microscopic dynamics.

The above scenario is confirmed using (with some important changes) the method of Wolf et al. [20] for the computation of the Lyapunov exponent from numerical data. Basically, in presence of macroscopic chaos one observes that: the variables of the system are separated in slow variables (e.g. $m(t), \sigma(t))$ and fast variables (see fig.s 5 and 7 ). For a discussion of the FSLE in systems with slow and fast dynamics see Ref. [21]. 
Let us stress that also with a naive numerical treatment of the one-body description one can observe a rather clear evidence for the two regimes (the microscopic and the macroscopic ones) characterized by two very different "Lyapunov exponent". But, as discussed in the Appendix, the problem of the choice of the norm (that is present also working with the histograms) gives some unavoidable troubles which do not allow for a detailed comparison with the other methods.

Finally, it is interesting to mention the case of "single-band" chaos (see ref. [2]), where one has irregular behaviour with some macroscopic coherence, but there is not a separation between the microscopic and the macroscopic scales. The microscopic Lyapunov exponent has the same value of the macroscopic one $\left(\lambda_{\text {macro }}=\lambda_{1}\right)$. This means that in this case - as fig. 9 shows $-\lambda(\delta)$ has a single plateau, extending over a range of scales larger than that of the standard chaos (fig. 6), so as to include the macroscopic scales. Let us remark that this case of "single-band" chaos is reminiscent of the two-dimensional turbulence [17], where the $\lambda(\delta)$ does not depend on $\delta$ in a very large range of values; that is, a broad range of scales have equal characteristic times.

After the submission of this paper, the authors are informed of the recent preprint by $\mathrm{T}$. Shibata and K. Kaneko "Collective Chaos" (chao-dyn/9805009), where a related study is presented. In particular, T. Shibata and K. Kaneko use the FSLE to study the macroscopic properties of a heterogeneous GCM. Their results are in agreement with ours.

\section{ACKNOWLEDGMENTS}

We thank E. Aurell and L. Biferale for useful suggestions and a careful reading of the paper. This paper has been partially supported by INFM (PRA-TURBO) and MURST (program 9702265437).

\section{APPENDIX:}

One could naively think that the behaviour of $|\delta m(t)|$ and $\| \delta \rho_{t}||$ give the same information. In this appendix we show that the above idea does not work because of the unavoidable troubles related to the choice of a norm for $\delta \rho_{t}$.

In order to show this we discuss two explicit cases which clarify this point. As first example we consider $\rho(x)$ uniform in the interval $[a, b]$ and the perturbed distribution $\rho^{\prime}(x)$ uniform in the interval $[a+\delta m, b+\delta m]$, i.e. with a shifted average. Let us now compute two different norms of $\delta \rho=\rho^{\prime}-\rho$ :

$$
\begin{gathered}
\|\delta \rho\|_{1}=\int|\delta \rho(x)| \mathrm{d} x=\frac{2}{b-a}|\delta m| \\
\|\delta \rho\|_{2}=\sqrt{\int \delta \rho^{2}(x) \mathrm{d} x}=\sqrt{\frac{2}{(b-a)^{2}}|\delta m|} .
\end{gathered}
$$


Assuming that $\delta m$ is related to a GCM system, it is clear that the computation of the Lyapunov exponents according to the norms defined above gives, in this case (uniform distribution), two Lyapunov exponent different by a factor two. Nevertheless with different shape of the distribution we have different results. To show this last issue we consider now a $\rho(x)$ with a symmetric triangular shape in the interval $[a, b]$ and zero for $x<a$ or $x>b$. The perturbed $\rho^{\prime}$ has the same functional form with the average shifted of $\delta m$. The same computations as before lead to:

$$
\begin{gathered}
\|\delta \rho\|_{1}=(b-a)|\delta m|+O\left(|\delta m|^{2}\right) \\
\|\delta \rho\|_{2}=\sqrt{\frac{b-a}{2}}|\delta m|+O\left(|\delta m|^{2}\right) .
\end{gathered}
$$

From the above examples it is clear that the relation between $|\delta m|$ and $\|\delta \rho\|$ depends dramatically on the explicit norm used and on the shape of the $\rho$. In addition, in the real time evolution of $\delta \rho_{t}$ one has not only shifting of the average but also variations of $\sigma^{2}$ and other parameters of the distribution that complicate the interpretation of $\|\delta \rho\|$ in terms of $|\delta m|$. Therefore there is no reason to expect a quantitative agreement between numerical study (using histograms) of the one-body description and $|\delta m(t)|$. 


\section{REFERENCES}

[1] H. Chatè and P. Manneville, Prog. Theor. Phys. 87 (1992) 1.

[2] K. Kaneko, Phys. Rev. Lett. 65 (1990) 1391;

K. Kaneko, Physica D 55 (1992) 368;

K. Kaneko, Physica D 86 (1995) 158.

[3] A. Crisanti, M. Falcioni and A. Vulpiani, Phys. Rev. Lett. 76 (1996) 612.

[4] K. Kaneko, Phys. Rev. Lett. 63 (1989) 219;

K. Kaneko, Physica D 41 (1990) 38;

K. Kaneko, Phys. Rev. Lett. 65 (1990) 1391.

[5] T. Konishi and K. Kaneko, Jour. Phys. A 25 (1992) 6287.

[6] A.S. Pikovsky and J. Kurths, Phys. Rev. Lett. 72, (1994) 1644;

A.S. Pikovsky and J. Kurths, Physica D 76 (1994) 411.

[7] T. Shibata and K. Kaneko, Europhys. Lett. 38 (1997) 417

[8] G. Perez and H.A. Cerdeira. Phys. Rev. A 46 (1992) 7492.

[9] A.T. Winfree . The Geometry of Biological Times. (Springer-Verlag, New York 1980).

[10] A. Kolmogorov and S. Fomine, Eléments de la théorie des functions et de l' analyse functionnelle, chap. III. (Editions MIR, Moscow)

[11] A.J. Kreuzer, Nonequilibrium thermodynamics and its statistical foundations. (Clarendon Press, Oxford 1981).

[12] G. Benettin, L. Galgani, A. Giorgilli and J.M. Strelcyn, Meccanica 15 (1980) 9.

[13] G. Paladin and A. Vulpiani, Phys. Rep. 156 (1987) 147.

[14] J.P. Eckmann and I. Procaccia, Phys. Rev A 34 (1986) 659.

[15] J. Kurths and A. Brandenburg,Phys. Rev A 44 (1991) 3427.

[16] E. Aurell, G. Boffetta, A. Crisanti, G. Paladin and A. Vulpiani, Phys. Rev. Lett. 77 (1996) 1262.

[17] E. Aurell, G. Boffetta, A. Crisanti, G. Paladin and A. Vulpiani, J. Phys. A 30 (1997) 1.

[18] V. Artale, G. Boffetta, A. Celani, M. Cencini and A. Vulpiani, Phys. of Fluids. 9 (1997) 3162.

[19] G. Boffetta, A. Crisanti, F. Paparella, A. Provenzale and A. Vulpiani, "Slow and fast dynamics in coupled systems: a time series analysis view" . chao-dyn/9709014 Physica $D$ in press.

[20] A. Wolf, J.B. Swift, H.L. Swinney and J.A. Vastano, Physica D 16 (1985) 285.

[21] G. Boffetta, P. Giuliani, G. Paladin and A. Vulpiani, "An extension of the Lyapunov analysis for the predictability problem". chao-dyn/9801030, Jour. Atm. Science in press. 


\section{FIGURE CAPTIONS}

FIGURE 1: (a) Plot of $m(t)$ versus $m(t-1)$ for the system (11) with $a=1.7 \epsilon=0.3$ and $N=10^{6}$; fig (1b) is an enlargement of (a).

FIGURE 2: Probability distribution function $P_{n}(x)$ for two different $x_{n}$ for the system eq. (11) with $a=1.8, \epsilon=0.3$ and $N=10^{5}$. The distribution are computed following $x_{n}(t)$ for $5 \cdot 10^{5}$ temporal steps.

FIGURE 3: Absolute value of the correlation function $C_{\text {macro }}(\tau)$ (solid line) compared with the absolute value of the correlation functions for two different $x_{i}(t), C_{\text {micro }}^{i}(\tau)$, (dashed and dot-dashed lines). The parameters are chosen as in fig. 2.

FIGURE 4: $<\ln |\delta m|>$ versus $t$ for the system (11) with $a=1.8, \epsilon=0.08$ and $N=10^{4}$. The squares are for an initial perturbation $\delta m(0)=10^{-6} \ll 1 / \sqrt{N}$, the circles for $\delta m(0)=0.15 \gg 1 / \sqrt{N}$, and the dotted line has slope $\lambda_{\text {micro }}$. The average is over $10^{3}$ realizations.

FIGURE 5a: $\lambda(\delta)$ versus $\delta$ for the system (1) with $a=1.7, \epsilon=0.3$ for $N=10^{4}(\times)$, $N=10^{5}(\square), N=10^{6}(\odot)$ and $N=10^{7}(\triangle)$. The first plateau corresponds to the microscopic Lyapunov exponent $\lambda_{\text {micro }} \approx 0.17$ and the second one to the macroscopic Lyapunov exponent $\lambda_{\text {macro }} \approx 0.007$. The average is over $2 \cdot 10^{3}$ realizations for $N=$ $10^{4}, 10^{5}, 10^{6}$ and 250 realizations for $N=10^{7}$. The system has been initialized in order to leave the attractor unchanged (as explained in the text).

FIGURE 5b: The same as figure 5a rescaling the $\delta$-axis with $\sqrt{N}$.

FIGURE 6: $\lambda(\delta)$ versus $\delta$ for the system (1) with $a=1.8, \epsilon=0.08$ for $N=10^{4}(\square)$, $N=10^{5}(\odot)$ and $N=10^{6}(\triangle)$. The plateau corresponds to the microscopic Lyapunov exponent $\lambda_{\text {micro }} \approx 0.5$. The average is over $2 \cdot 10^{3}$ realizations.

FIGURE 7: $<\ln |\delta m|>$ versus $t$ for the system (11) with $a=1.7, \epsilon=0.3$ and $N=10^{4}$ (upper curve), $N=10^{5}$ (middle curve), $N=10^{6}$ (lower curve) The straight lines correspond to the two exponential growth the microscopic one $\left(\lambda_{\text {micro }} \approx 0.17\right)$ and the macroscopic $\left(\lambda_{\text {macro }} \approx 0.01\right)$. The average is over 500 realizations.

FIGURE 8: $\Gamma(\tau)$ versus $\tau$ for the system (11) with $a=1.7, \epsilon=0.3$ and $\mathcal{A}=250$, the dashed line corresponds to $\lambda_{\text {macro }} \approx 0.006$. The distance has been plotted every two time steps to eliminate oscillations given by the swiching between bands.

FIGURE 9: $\lambda(\delta)$ versus $\delta$ for the system (1) with $a=1.5, \epsilon=0.3$ for $N=10^{4}(\square)$, $N=10^{5}(\odot)$. The plateau corresponds to a Lyapunov exponent $\lambda_{\text {micro }} \approx 0.28$. The average is over $2 \cdot 10^{3}$ realizations. 


\section{FIGURES}
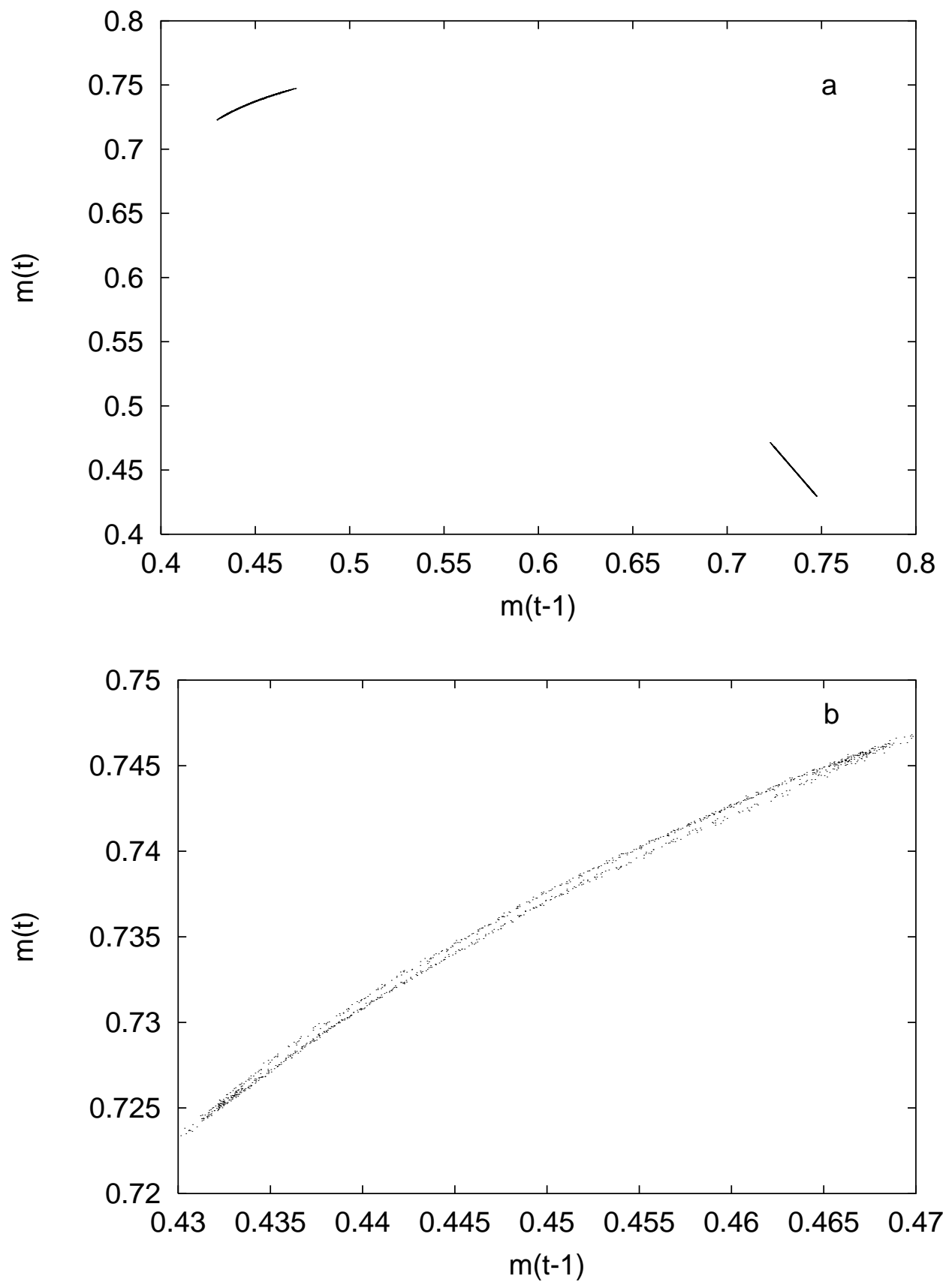

FIG. 1. 


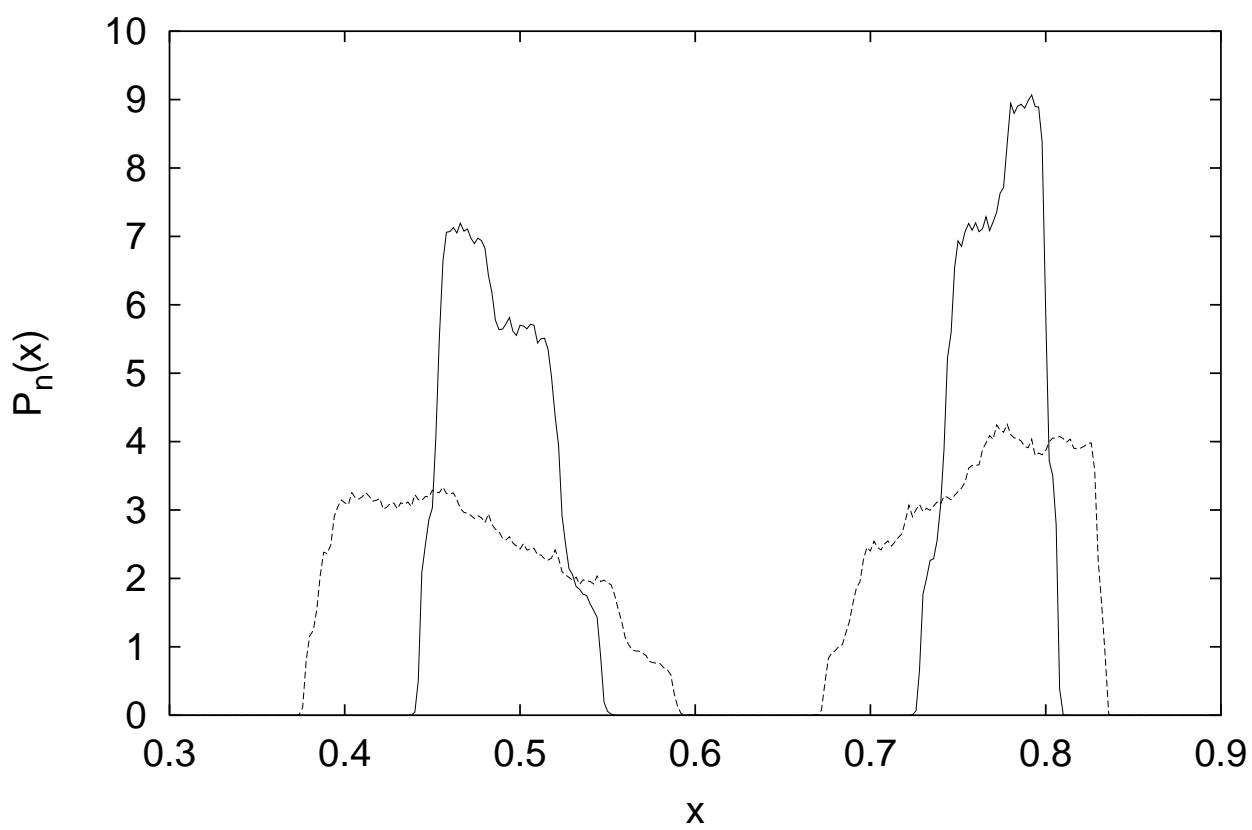

FIG. 2. 


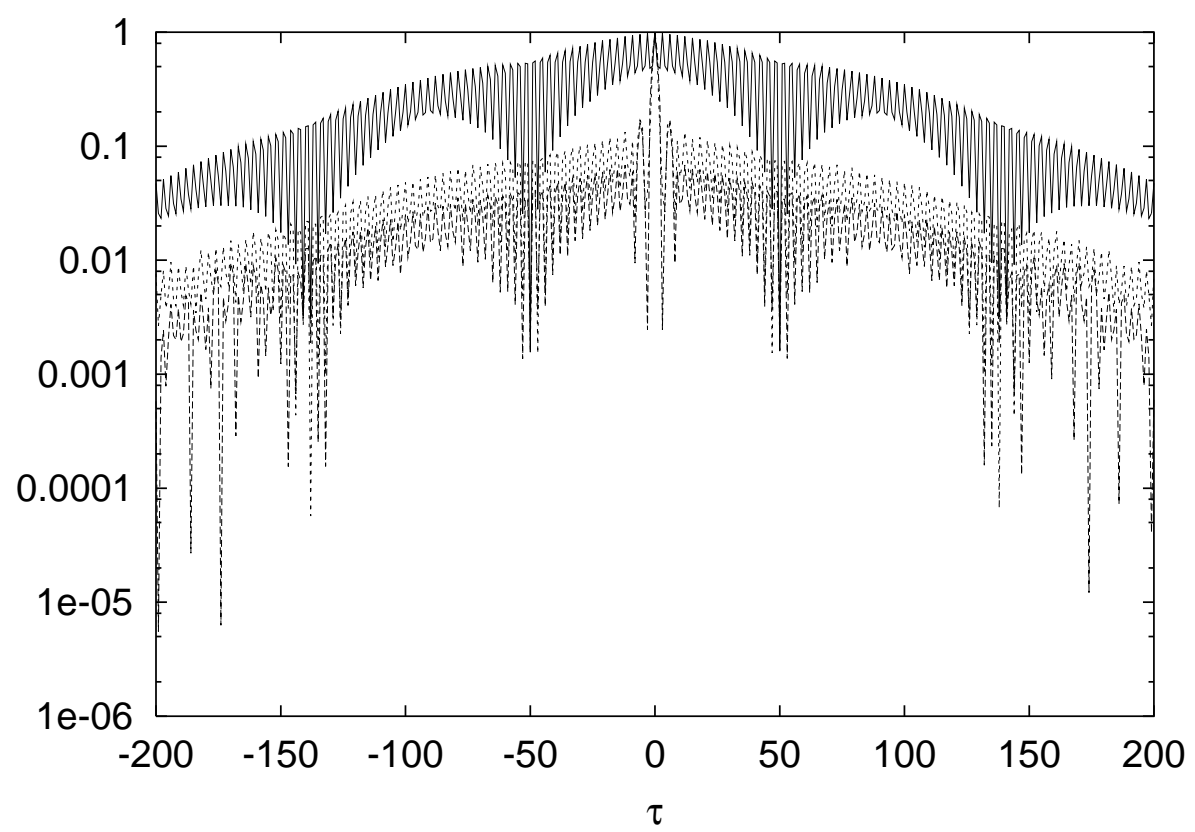

FIG. 3. 


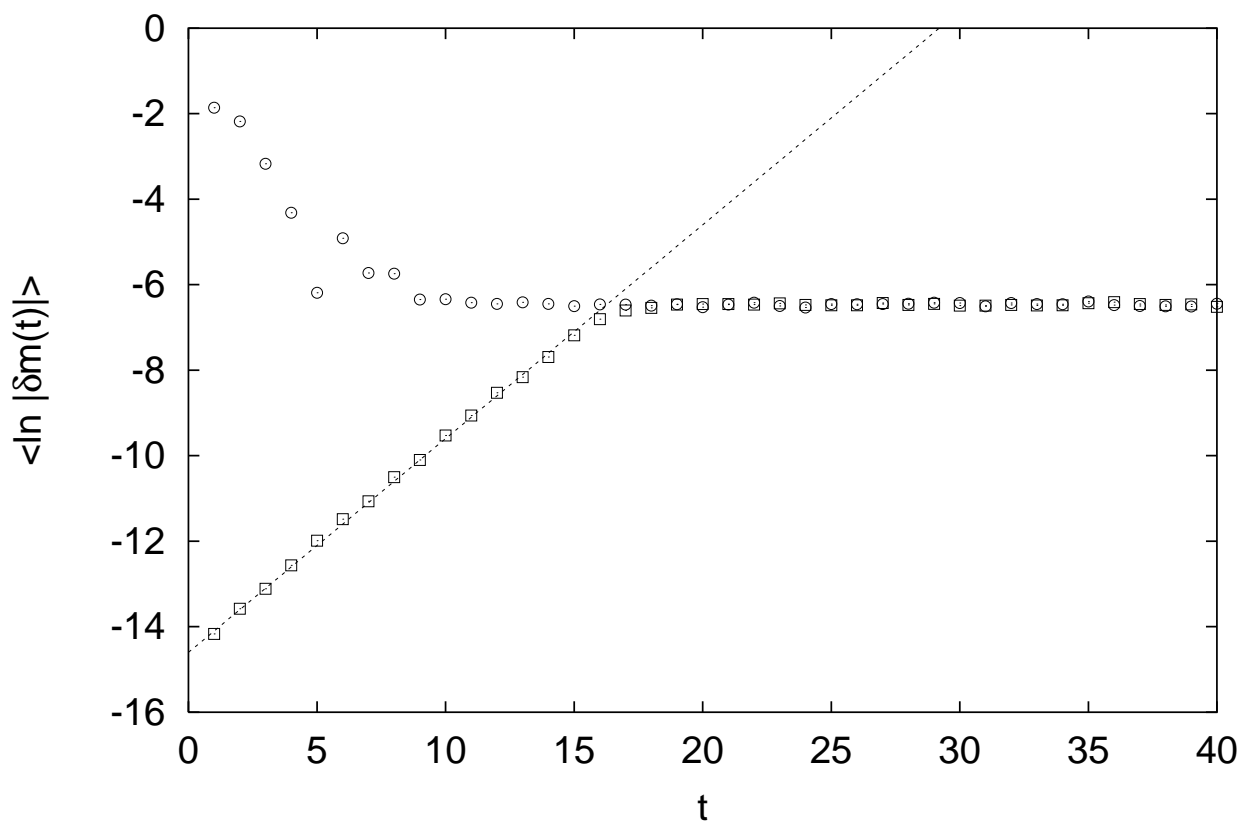

FIG. 4. 

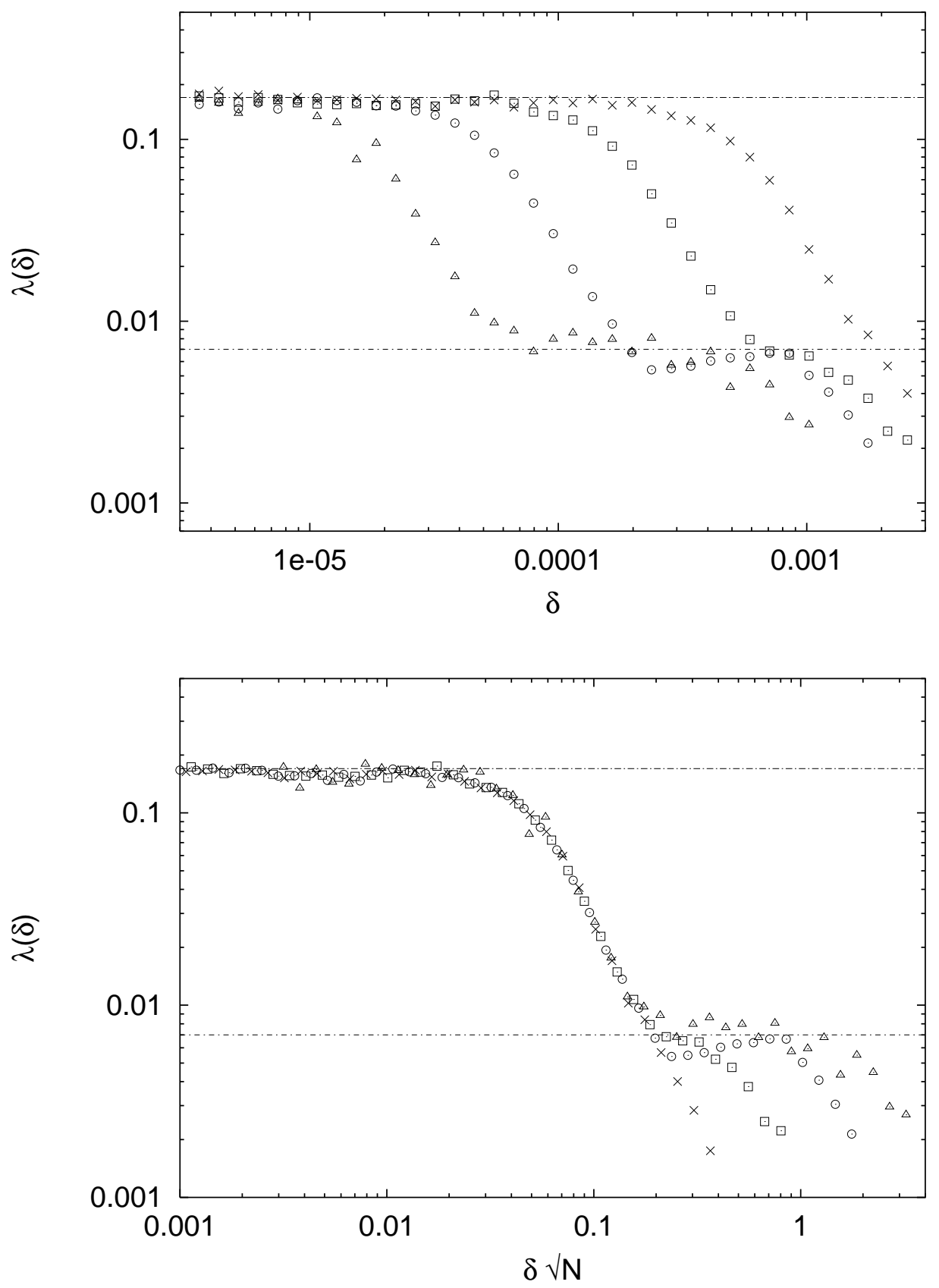

FIG. 5. 


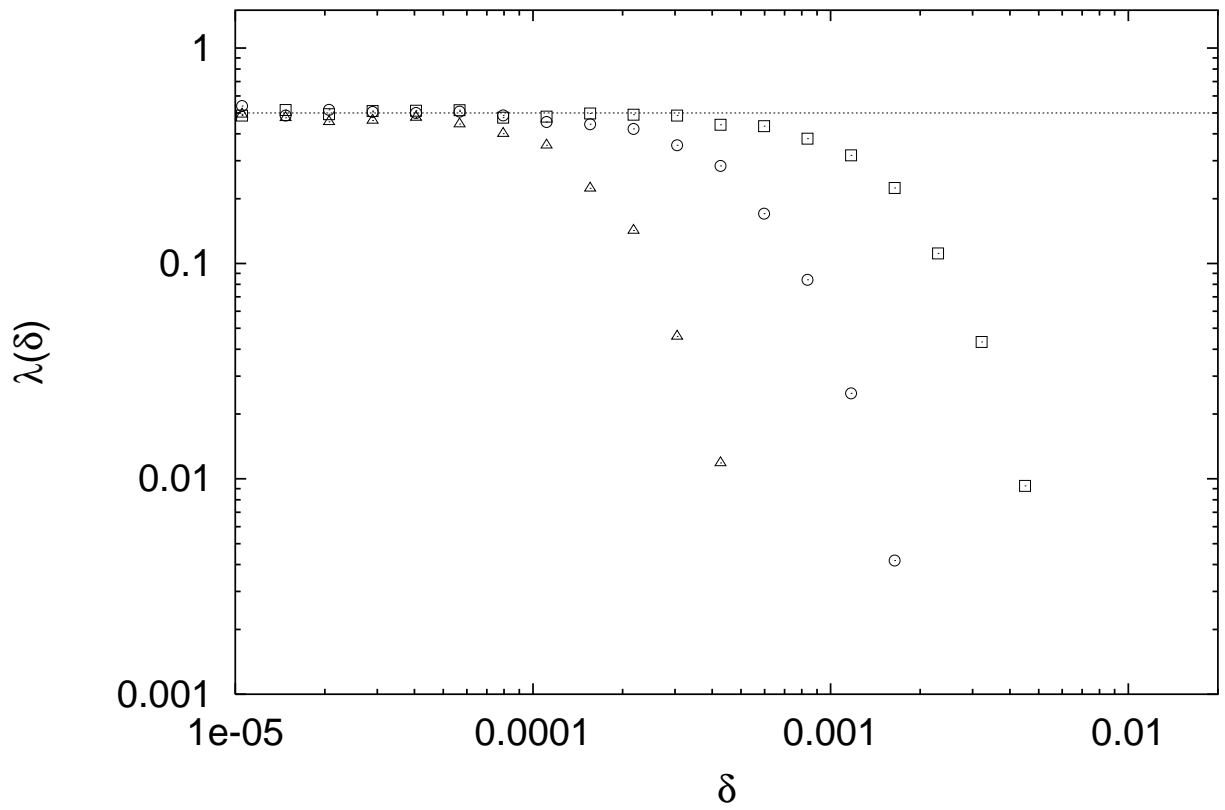

FIG. 6. 


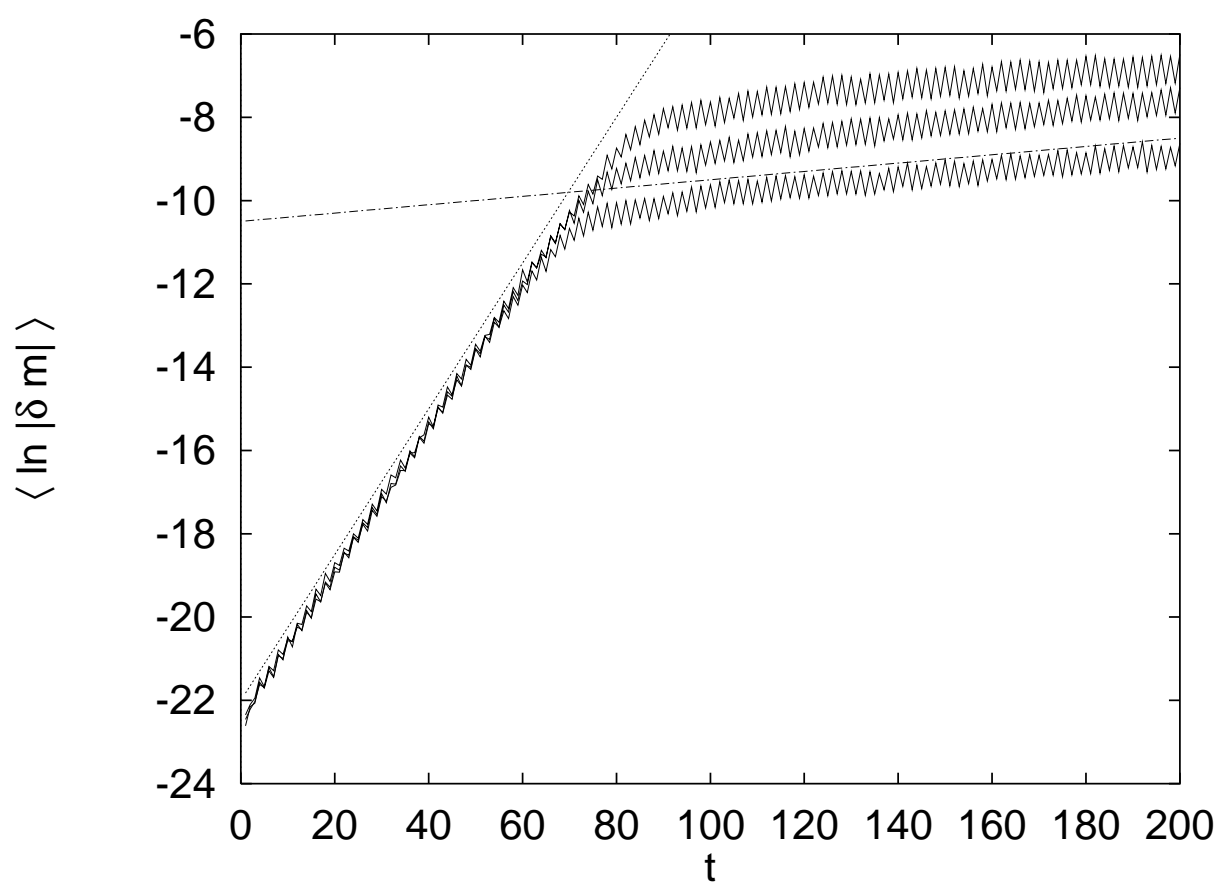

FIG. 7. 


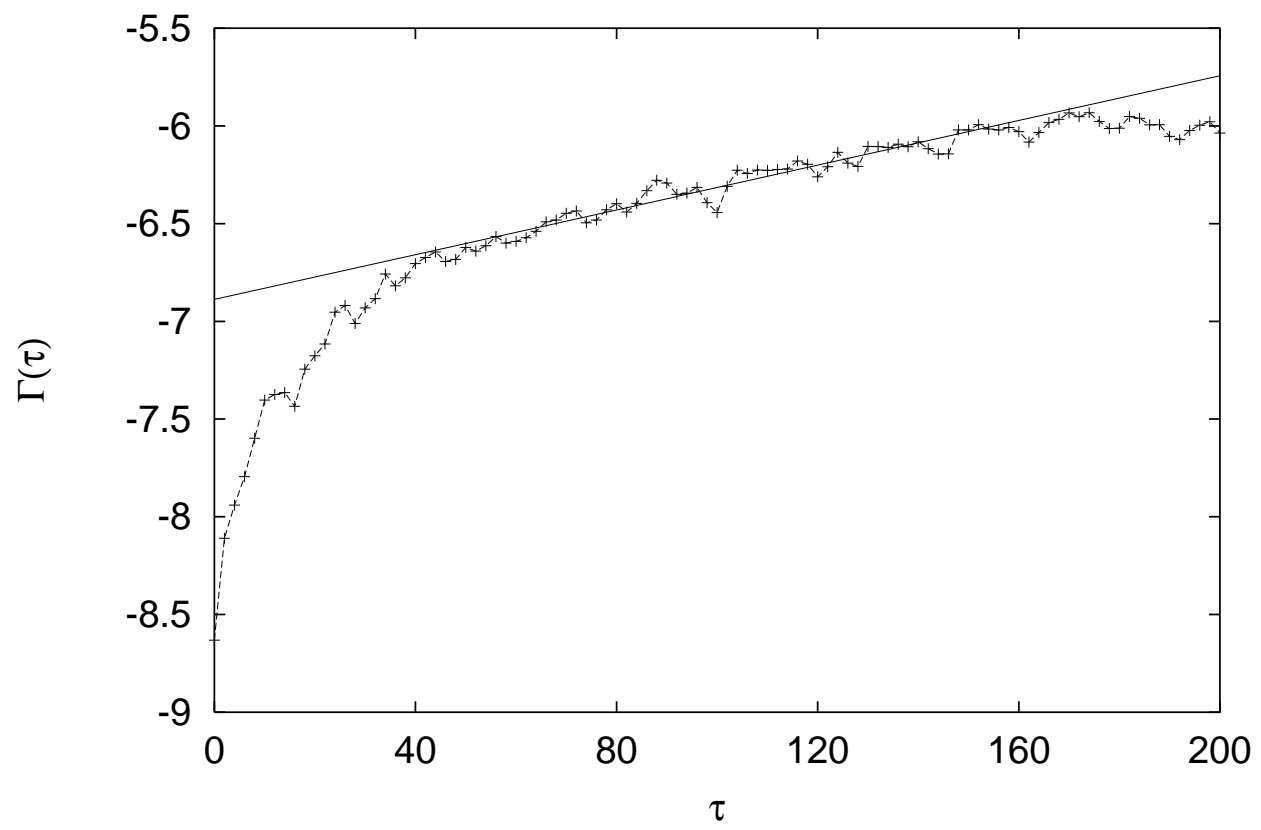

FIG. 8 . 


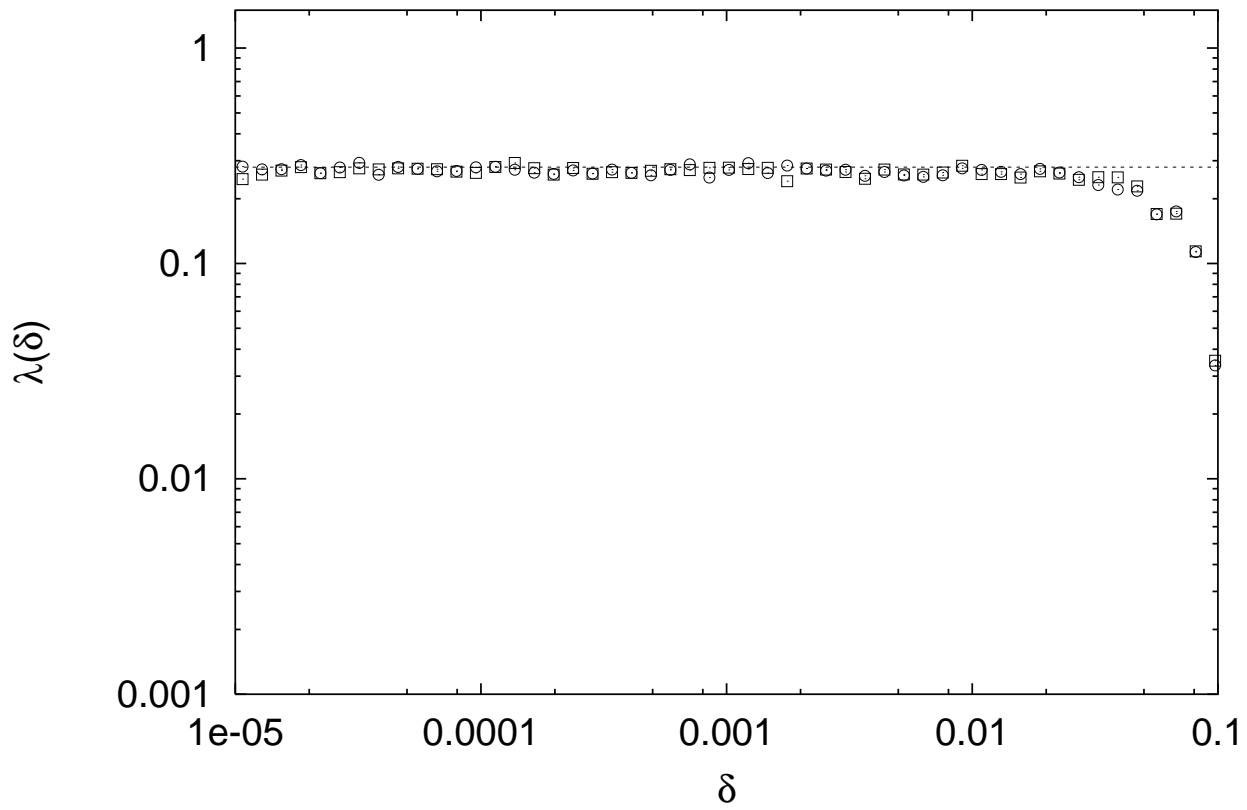

FIG. 9 